\title{
Goethe, le roman et la nouvelle : le cas des Affinités électives
}

\section{François Genton}

\section{OpenEdition}

\section{Journals}

Édition électronique

URL : http://journals.openedition.org/cei/177

DOI : $10.4000 /$ cei. 177

ISSN : 2260-779X

\section{Éditeur}

UGA Éditions/Université Grenoble Alpes

\section{Édition imprimée}

Date de publication : 15 mars 2010

Pagination : 181-191

ISBN : $978-2-84310-164-9$

ISSN : 1770-9571

\section{Référence électronique}

François Genton, "Goethe, le roman et la nouvelle : le cas des Affinités électives », Cahiers d'études italiennes [En ligne], 10 | 2010, mis en ligne le 15 septembre 2011, consulté le 27 mars 2021. URL http://journals.openedition.org/cei/177 ; DOI : https://doi.org/10.4000/cei.177 


\title{
GOETHE, LE ROMAN ET LA NOUVELLE : LE CAS DES AFFINITÉS ÉLECTIVES
}

\author{
François Genton \\ Université Stendhal - Grenoble 3
}

Les historiens de la littérature datent du Décaméron ${ }^{\mathrm{I}}$, sinon la naissance, du moins la percée en Italie, puis en Europe du genre de la nouvelle en prose et en langue vernaculaire. Le mot «nouvelle» s'impose en Allemagne vers 1476 , dans une première traduction de Boccace, dont le long titre joue explicitement sur trois langues : d'abord le grec (Decamerone), puis l'italien (welsch) qui permet d'introduire le nom d'un genre "exotique» (cento novelle), enfin l'allemand qui traduit abondamment les deux expressions précédentes par "cent histoires ou fables nouvelles ${ }^{2}$ ». Un genre exotique, que la littérature allemande a tardé à adopter. Les Nouvelles exemplaires de Cervantes ne sont traduites, à partir de plusieurs versions françaises, qu'en $1753^{3}$ et en 1779 seulement Heinrich von Soden fait paraître à Leipzig un texte allemand directement traduit de l'original espagnol ${ }^{4}$. En I8oI, une nouvelle version de Dietrich Wilhelm Soltau a inspiré directement Hoffmann dont le récit Les dernières aventures du chien Berganza, dans les Fantaisies à la manière de Jacques Callot, est imité de la nouvelle de Cervantes Le Colloque des chiens.

I. Pour la commodité de la lecture nous citons les titres français usuels ou parfois traduits par nous des œuvres étrangères, nous contentant de renvoyer en note au titre original. Sauf indication contraire, nous avons traduit les citations allemandes.

2. Voici le titre complet indiqué par la bibliothèque de l'université de Göttingen : Hie hebt sich an das puch von seinem meister In greckisch genant decafmeron/ daz ist cento nouelle in welsch Vun hundert histori oder neue fabel in teutsche/ die der hoch gelehrte poete Johannes boccacio ze liebe und früntschafft schreibet. Geendet seliglichen zu Ulm (Joh. Zeiner), [environ I476].

3. Satyrische und lehrreiche Erzehlungen des Michel de Cervantes Saavedra, Verfasser der Geschichte des Don Quischotts, nebst dem Leben dieses berühmten Schriftstellers, traduit (du français) par J. C. Conradi, Francfort et Leipzig, I753.

4. Moralische Erzählungen des Miguel de Cervantes, Verfasser des Don Quixote, Königsberg, I8oI. 
Vers i80o la référence espagnole renforce les références anglaise et italienne bien plus anciennes pour saper la prépondérance de la littérature française dans tous les genres littéraires. Dès les dernières décennies de l'Ancien Régime le répertoire "national» des Lessing, Goethe et Schiller s'affirme contre les théâtres français des cours, qui disparaissent peu avant la Révolution française, et la poésie allemande a abandonné l'alexandrin pour des rythmes plus libres, inspirés de la métrique grecque et latine, et dans le genre narratif le succès international des Souffrances du jeune Werther (1774) du jeune Goethe signale l'irruption du roman allemand dans l'avant-garde littéraire européenne. À l'instar de l'évolution française et anglaise, l'art du récit se caractérise par une intense fermentation en Allemagne. Une partie importante du public cultivé prend immédiatement connaissance des dernières productions françaises et anglaises qui sont très souvent traduites, parfois à plusieurs reprises, en allemand, et sous l'effet de cette double pression du goût anglais et français du public et de la forte présence des traductions, les auteurs allemands tentent de prouver qu'eux aussi peuvent contribuer à renouveler le genre narratif. La nouvelle allemande est née dans ce contexte historique. La première définition "classique» du genre est donnée en 1772 par Christoph Martin Wieland, dans une note de la deuxième édition de son roman $L a$ victoire de la nature contre l'exaltation ou les aventures de Don Sylvio de Rosalva ${ }^{5}$ un roman dont le héros est aussi fasciné par les contes de fées que Don Quichotte l'est par Amadis de Gaule. L'histoire grotesque du prince Biribinker parvient à le guérir, c'est-à-dire à le ramener à la réalité, dans l'esprit du siècle des Lumières contre le goût galant des contes merveilleux qui passent aussi pour courtois :

Nouvelle est un terme par lequel on désigne de préférence des récits qui se distinguent des grands romans par la simplicité du plan et la dimension modeste de la fable se rapportant à ce dernier genre comme les saynètes se rapportent aux grands genres de la tragédie et de la comédie. Les Espagnols et les Italiens en possèdent un grand nombre 6 .

Notons que la nouvelle reste ici un genre espagnol et italien, sans autre caractéristique que la simplicité et la brièveté. Goethe, qui avait renouvelé

5. Nous avons traduit le titre allemand Der Sieg der Natur über die Schwärmerey oder die Abentheuer des Don Sylvio von Rosalva. La première édition ( 1764 ) avait été traduite en français dès 1760 sous le titre suivant : Les Avantures merveilleuses de Don Sylvio de Rosalva.

6. «Novellen werden vorzüglich eine Art von Erzählungen genannt, welche sich von den großen Romanen durch die Simplizität des Planes und den kleinen Umfang der Fabel unterscheiden, oder sich zu denselben verhalten wie die kleinen Schauspiele zu den großen Tragödie und Komödie. Die Spanier und Italiener haben deren eine unendliche Menge.» (Cité par Herbert Krämer, Theorie der Novelle, Stuttgart, Reclam, 1976, p. 9.) 
le roman épistolaire avec Les Souffrances du jeune Werther, renoue avec les grandes formes narratives à l'époque de la Révolution française : de 1793 à 1797 il publie deux épopées Le Renard (d'après Le Roman de Renart), Hermann et Dorothée, le cycle de nouvelles Entretiens d'émigrés allemands et les Années d'apprentissage de Wilhelm Meister. Difficile de ne pas faire le lien entre cette fécondité narrative et l'évolution du poète face aux bouleversements de la Révolution française. Avant ces récits, Goethe avait écrit trois pièces antirévolutionnaires, une sorte de réaction épidermique dénonçant aussi bien une certaine décadence de la noblesse française que l'absurdité (selon Goethe) de la représentation d'un gouvernement populaire. À la différence de l'épopée animale, qui présente un tableau sans illusions des intrigues courtisanes, Hermann et Dorothée, Les Entretiens et Wilhelm Meister ont en commun de présenter un contenu positif, à savoir l'idée d'une victoire toujours possible de la mesure sur les passions, de la communication sur la violence, du sentiment authentique et de la valeur personnelle sur les préjugés sociaux. L'épopée Hermann et Dorothée finit bien, par l'union des héros malgré les obstacles familiaux et sociaux. Dans Les Entretiens d'émigrés allemands une baronne, ses amis, ses enfants et ses domestiques, sont contraints de fuir les contrées allemandes envahies par les troupes françaises. Le jeune cousin Karl, partisan passionné de la Révolution, menace de causer une grave mésentente. La baronne sauve alors la cohésion de la petite société en édictant la règle d'une sociabilité attentionnée (gesellige Schonung) qui n'est autre qu'une pédagogie par le récit, favorisant l'expression des uns, l'écoute des autres et la communication de tous. Trois personnages racontent sept histoires au cours d'une journée. Le récit-cadre, on l'aura compris, est imité du Décaméron, les récits sont tirés d'anecdotes, de souvenirs, des Mémoires du Maréchal de Bassompierre (1665) et des Cent nouvelles nouvelles (I460). Seuls deux d'entre eux sont vraiment de l'invention de Goethe, dont le Conte (Das Märchen). Le roman Les années d'apprentissage de Wilhelm Meister, qui fut considéré dès sa parution comme le premier roman allemand moderne et fut défini en I8I9 comme le protopype du Bildungsroman ${ }^{7}$, mêle tous les genres littéraires : la poésie, le théâtre (qui est la première passion du héros)

7. La notion fut lancée en I8I9 dans un exposé intitulé "De l'essence du roman de formation " (Über das Wesen des Bildungsromans») par Johann Carl Ludwig Morgenstern, professeur à l'université de Dorpat (aujourd'hui Tartu), en Estonie, alors sous domination russe. L'idée du roman moderne en tant que description d'un monde prosaïque, mais raisonnable, auquel l'individu apprend à s'adapter au cours d'un long apprentissage est au centre des développements de Hegel dans ses cours d'esthétique (I8I7-I829), dont les textes ont été rassemblés et édités par H. G. Hotho en I835, cinq ans après la mort de son maitre. Voir par exemple le chapitre sur la dissolution du romanesque dans G. W. F. Hegel, Ästhetik I/II, Stuttgart, Reclam, p. 609. 
et plusieurs genres de récits, dont l'autobiographie piétiste au Livre VI («Les confessions d'une belle âme», d'après les entretiens du jeune Goethe avec Susanna von Klettenberg). La nouvelle n'apparaît pas comme une forme bien définie dans ces récits longs et brefs de la dernière décennie du $\mathrm{XVIII}^{\mathrm{e}}$ siècle, mais une volonté de varier les genres, de les confronter, une propension à la mise en miroir, dont la Baronne des Entretiens énonce le principe : "J'aime les histoires parallèles. L'une renvoie à l'autre et explique sa signification mieux que d'arides discours ${ }^{8}$.» Pour le moment, l'écriture nouvellistique semble se borner à la technique du collage, de la mise en miroir et de la fragmentation du récit : la nouvelle en tant que genre y a peu de part.

En I809 paraît le roman les Affinités électives. Il a été conçu au départ comme une nouvelle destinée à être insérée dans Les Années de voyage de Wilhelm Meister, roman dont la version définitive est publiée en I829, huit ans après la première édition. Édouard (qui s'est appelé Otto) et Charlotte vivent sur leurs terres et goûtent le plaisir d'être enfin unis, après deux premières unions malheureuses. Ils accueillent Otto, l'ami d'Édouard (Eduard), et Odile (Ottilie), la nièce de Charlotte. Le phénomène chimique des "affinités électives", dont les amis ont plaisanté, bouleverse leurs vies : Édouard et Odile, Charlotte et Otto sont irrésistiblement attirés les uns vers les autres, et l'enfant que Charlotte attend d'Édouard a les yeux d'Odile et le visage d'Otto. Cet enfant, prénommé Otto, se noie par la faute d'Odile, cette dernière renonce à son amour et se laisse mourir de faim, Édouard la suit dans la tombe. L'intrigue est riche en détails symboliques qu'il est impossible de citer ici. Il suffira de mentionner que cette petite société, qui travaille à façonner son environnement bâti et non bâti, se montre incapable de façonner son destin et d'affronter la vie. Le seul personnage véritablement "fort» n'est autre qu'Odile, dont le refus ascétique de la vie, un trait constant, entraîne les catastrophes finales. Alors que Charlotte avait fait aplanir le petit cimetière du domaine et apposer les pierres tombales au mur de la chapelle, c'est la mort qui triomphe d'une petite aristocratie campagnarde sans autre avenir qu'une dévotion mortuaire un peu ridicule autour de la tombe d'une Odile érigée en sainte à la fin du roman. Ce tournant "catholique», incarné par le bas peuple et un artiste, peut être compris comme la critique voilée d'une tendance

8. "Ich liebe mir sehr Parallelgeschichten. Eine deutet auf die andere hin und erklärt ihren Sinn besser als viele trockene Worte." (J. W. von Goethe, "Unterhaltungen deutscher Ausgewanderten ", Sämtliche Werke, Munich et Zurich, t. 9, p. 345.) 
propre au romantisme allemand, tout aussi illusoire que l'agitation oisive d'une classe qui n'a plus de raison d'être.

C'est à des personnages anglais qu'il revient de formuler une vision de l'humanité plus positive et plus moderne, à propos de la nouvelle «Les jeunes voisins singuliers» : une petite fille se querelle si fort avec son jeune voisin que les deux enfants doivent être séparés. Quelques années plus tard, le garçon, désormais un jeune homme, est invité au mariage de sa voisine. Il pilote le bateau qui porte sur un fleuve les promis et leurs amis. La jeune fille saute à l'eau, il plonge dans le fleuve pour la sauver. La jeune évanouie est ramenée à la rive, dénudée, séchée. Les jeunes gens revêtent les habits de noce d'un jeune couple et rejoignent leurs familles et leurs amis. L'Anglais qui rapporte cette histoire accompagne un Lord qui préfere à la vie de château (celle des personnages allemands) une errance plus économique et plus instructive : les deux personnages, équipés des dernières inventions techniques, étudient le monde. Rien ne leur échappe, pas même les richesses minérales enfouies dans le sol dont la présence cause de si douloureuses migraines à Odile. Autrement dit, si les Anglais incarnent la rationalité technique de la patrie de la révolution industrielle et du capitalisme moderne, Odile représente un inconscient mortifère auquel répugne une exploitation rationnelle des ressources naturelles. Walter Benjamin a parlé à propos de ce texte d'une «nouvelle-type»(Musternovelle), voire d'une nouvelle "exemplaire9" du roman comme la vie à la mort, l'amour vécu à l'amour fantasmé, le bonheur à la béatitude, le fleuve impétueux, mais dominé, à l'eau calme, mais profonde et meurtrière, du lac du grand domaine, l'énergie vitale du héros à la passivité criminelle d'Ottilie. La société des Affinités électives n'est pas faite pour l'idéal de communication ouverte propre à celle des Entretiens, et le narrateur ne parvient pas à détendre l'atmosphère qui règne dans le château de Charlotte. Il est vrai que cette dernière a deviné que l'«événement» (Begebenheit) rapporté innocemment par l'Anglais a concerné son ami Otto, dont on comprend qu'il fut le fiancé malheureux de l'héroöne de la nouvelle. C'est cet événement «nouveau» qui justifie la désignation générique de "nouvelle». Goethe donne en I827 une définition du genre ( $u n$ événement inouï qui s'est passé $\left.{ }^{10} »\right)$ en variant une

9. Walter Benjamin, "Goethes Wahlverwandtschaften", Illuminationen. Ausgewählte Schriften I, Francfort, Suhrkamp, I977, p. I04 et suiv. Ce texte, rédigé en I92I-I922, a été publié pour la première fois par Hugo von Hofmannsthal dans les Neue deutsche Beiträge, 2 vol., I925, I, p. 83-138; 2, p. I34-I68.

Io. "[...] denn was ist Novelle anders als eine sich ereignete, unerhörte Begebenheit." (Johann Peter Eckermann, Gespräche mit Goethe in den letzten Jahren seines Lebens, Francfort, Insel, 1981, p. 207 et suiv. [2I janvier I827].) 
expression que l'on trouve à un point central du récit qu'il publie l'année suivante sous le titre de Nouvelle ("l'événement étrange, inouï ${ }^{\mathrm{II}}$ ). Dans Les Affinités électives, la nouvelle décrit un couple qui affirme librement son désir face aux contraintes multiples du monde - alors que les personnages du roman, en conflit apparent avec leurs désirs, se laissent entraîner dans une spirale mortifere qui n'est autre que l'expression de la pulsion de mort d'Odile. La religiosité et le conformisme morbides d'une part, la sottise et l'inanité des plaisirs d'une aristocratie sans inspiration de l'autre sont opposés à la modernité, d'une part l'esprit pratique et scientifique des Anglais, de l'autre la liberté morale et sexuelle du jeune couple de la nouvelle. Aux personnages du roman s'opposent ceux de la nouvelle, et les Anglais font le lien entre les deux univers. La nouvelle esquisse le monde nouveau qui pourra se substituer à cet Ancien Régime moribond qu'incarnent les personnages du roman, et l'accomplissement personnel remplace la répression du désir : la nouvelle prosaïque dément l'élégie romanesque (et romantique).

Auteur en I8OI d'un texte sur Boccace ${ }^{12}$, Friedrich Schlegel formule une conception romantique de la nouvelle conçue comme le produit d'une tension entre la subjectivité de l'auteur et l'objectivité de la fable : la fable a au fond moins d'importance que la manière de la rapporter. Goethe n'a pas approuvé cette insistance sur la dimension subjective du genre et, malgré l'attirance qu'il éprouve pour le merveilleux et les sciences occultes, il produit des nouvelles qui sont aussi éloignées du merveilleux romantique des contes du jeune Tieck que de la tension et de la concision exacerbées de la prose narrative de Kleist, de même qu'il a rejeté explicitement les récits fantastiques de Hoffmann, c'est-à-dire l'intrusion disharmonique du merveilleux dans le réel ${ }^{13}$. La théorie romantique de la nouvelle met cependant en avant deux traits que Goethe a intégrés implicitement à sa conception du genre : comme Schlegel, il pense que la nouvelle est un genre adapté à la «société raffinée des gens de condition» (die feine Gesellschaft der edlern

II. "[...] dem seltsamen, unerhörten Ereignis [...]» (J. W. von Goethe, «Novelle», op. cit., [note 8], p. 448). I2. "Nachricht von den poetischen Werken des Johannes Boccaccio", Kritische Friedrich-Schlegel-Ausgabe, éd. E. Behler, J.-J. Anstett et H. Eichner, 1967, t. 2, p. 373-396. Nous citons aussi d'après l'édition des CEuvres complètes consultables sur internet: Friedrich Schlegel, Sämmtliche Werke, Vienne, I825, t. Io p. 4-36, disponible sur <http://books.google.fr/books?id=cx8QAAAAYAAJ\&pg=PT4I\&lpg=PT4I\&dq=\%22die+feine+Gesellsc haft+der+edlern+Stände\%22\&source=bl\&ots=6DtSJ 2 Rzrg\&sig=HLhp8HbWa9DGeTp-sNu_solFvo\&hl=fr\& ei=m5phSrTeEsqKnQPi_8T4Dw\&sa=X\&oi=book_result\&ct=result\&resnum=I> [consulté le I7 juillet 2009].

I3. Le 25 décembre I827 Goethe traduit en les approuvant de larges extraits d'un texte intitulé "On the Supernatural in Fictitious Compositions" (The Foreign Quarterly Review, juillet 1827) dans lequel Walter Scott polémise contre E.T.A. Hoffmann et le fantastique que lui aurait inspiré son "cerveau malade». Voir J. W. von Goethe, op. cit., [note 8], t. I4, p. 927-930. 
Stände ${ }^{\mathrm{I}}$ ) et se caractérise sur le plan structurel par une péripétie centrale («l'événement inouï»), un tournant, par exemple le saut dans le fleuve des deux personnages de la nouvelle insérée dans les Affinités électives. Cet élément du tournant (Wendepunkt) apparaît explicitement chez les romantiques allemands, ainsi en I803-I804 chez August Wilhelm Schlegel : «[...] la nouvelle exige des tournants décisifs, si bien que les parties principales du récit frappent bien le regard, et le drame a aussi cette exigence ${ }^{15}$.» Cette idée a été reprise bien plus tard, en I829, par Ludwig Tieck:

Bizarre, capricieuse, fantastique, légère et spirituelle, bavarde, se perdant dans la description de détails, tragique et comique, profonde et taquine, la vraie nouvelle supporte toutes ces nuances et ces caractéristiques, il lui faudra seulement ce tournant singulier qui attire l'attention et la distingue de tous les autres genres de récit ${ }^{16}$.

Le récit de Goethe intitulé Nouvelle se déroule dans le cadre d'une petite principauté dont le souverain est parti à la chasse, tandis que son épouse se promène dans les ruines d'un château médiéval. Le feu se déclare sur le marché de la petite ville, un tigre et un lion s'échappent d'une ménagerie. Honorio, le jeune homme chargé d'accompagner la princesse tue le tigre, qui ne représentait aucun danger, car il était quasiment apprivoisé, comme le déplorent les propriétaires de la ménagerie, accourus pour réclamer qu'on laisse au moins la vie sauve au lion. Le prince leur accorde la permission de capturer le fauve : par la seule force de la musique et de la poésie, un petit garçon domine le roi des animaux. On pense au fameux poème didactique de Schiller intitulé Chant de la cloche (I799), dans lequel l'incendie symbolise la Révolution et l'incapacité du peuple à exercer une souveraineté raisonnée, ou à la ballade L'apprenti sorcier, écrite par Goethe en 1827 , où seul le maître est capable de rétablir l'ordre. Ici, le problème est examiné du point de vue du prince, dont l'intérêt est d'être proche de son peuple et de ne pas exercer ou faire exercer la violence quand d'autres moyens, plus doux et plus beaux, préservent la vie et la cohésion sociale. La mort du tigre est effacée par la survie du lion, Honorio, le fidèle mais trop rapide soldat, est invité à se maîtriser, et de toute évidence la douceur

I4. Ibid., p. 36 .

15. "[...] die Novelle bedarf entscheidender Wendepunkte, so daß die Hauptmassen der Geschichte deutlich in die Augen fallen, und dies Bedürfnis hat auch das Drama." (August-Wilhelm Schlegel [frère de Friedrich], "Über die Novelle», Vorlesungen über schöne Literatur und Kunst, III. Theil [1801/I802], cité par Herbert Krämer, [note 6], p. 19.)

I6. «Bizarr, eigensinnig, phantastisch, leicht witzig, geschwätzig und sich ganz in Darstellung auch von Nebensachen verlierend, tragisch wie komisch, tiefsinnig und neckisch, alle diese Farben und Charaktere läßt die echte Novelle zu, nur wird sie immer jenen sonderbaren auffallenden Wendepunkt haben, der sie von allen andern Gattungen der Erzählung unterscheidet.» ("Über die Novelle», I829, cité par Herbert Krämer, [note 6], p. 2I.) 
est un mode de gouvernement et de gestion de la nature plus sûr que la violence - et le maître ne peut se passer de l'enfant-poète. Une interprétation possible, parmi quantité d'autres : la révolution (l'incendie, les forces brutes de la nature) ne peut être réprimée par des moyens violents, mais prévenue et éventuellement contenue par une politique respectueuse de l'autre et qui sait gagner les âmes par les moyens de la beauté et de la poésie. Une utopie conservatrice associant l'élite, les artistes et le peuple dans une harmonie sans cesse renouvelée - et tenant compte des revendications libérales du XIX ${ }^{\mathrm{e}}$ siècle. Dans sa dernière grande ouvre épique, Les années de voyage de Wilhelm Meister (I82I-I829), Goethe a abondamment recouru aux techniques ici décrites, notamment au collage et au montage. Il est impossible d'entrer dans le détail de ce dernier roman qui est à lui seul une longue réflexion sur les perspectives d'une humanité où l'ère des révolutions politique et industrielle contraint à définir de nouveaux modes d'existence et d'organisation. Il suffira de dire que cette réflexion prolonge les éléments déjà présents dans les Affinités électives. La nouvelle, cet événement "inouï", bouscule les fausses certitudes et habitudes des personnages (ou des sociétés) qui suivent leurs passions, leurs pulsions et les conventions et non leur conscience, leur raison ou leur intuition. Elle se distingue du roman, sur le plan de l'intrigue, par l'unicité de sa ligne narrative et, sur le plan structurel, par l'élément du «tournant» (Tieck) ou de «l'événement inouï» (Goethe).

Ce modèle réaliste de l'anecdote significative s'est imposé dans les lettres allemandes et dans la théorie allemande de la nouvelle. En i87i, Paul Heyse, fameux nouvelliste allemand en son temps et Prix Nobel de littérature en I9IO, a formulé cette définition en se référant explicitement au modèle de Boccace (Décaméron, $\mathrm{V}^{\mathrm{e}}$ journée, $9^{\mathrm{e}}$ nouvelle) :

Même face à une matière des plus intérieures et des plus riches, le narrateur serait bien avisé de se poser d'abord la question de savoir "où est le faucon», ce trait spécifique qui distingue cette histoire de mille autres ${ }^{17}$.

Heyse se réfère ici au faucon qui est le seul bien que possède encore le chevalier Federigo degli Alberighi qui s'est ruiné pour la belle et riche veuve Giovanna. L'oiseau de chasse est sacrifié pour un repas préparé en l'honneur de la belle. En lui se condense toute la nouvelle : l'amour éperdu du chevalier, mais aussi la reconnaissance et la générosité de la belle qui

I7. «[...] [es könnte] nicht schaden, wenn der Erzähler auch bei dem innerlichsten oder reichsten Stoff sich zuerst fragen wollte, wo "der Falke“ sei, das Specifische, das diese Geschichte von tausend anderen unterscheidet.» (Paul Heyse, «Einleitung zu Deutscher Novellenschatz», I87I, cité par Herbert Krämer, [note 6], p. 4I.) 
finit par épouser cet homme ruiné et lui éviter la déchéance sociale, alors qu'elle vient de perdre son fils auquel elle voulait offrir ce faucon.

Quarante ans plus tard, en I9II, le jeune Georges Lukács assigne à la nouvelle la fonction de représenter la totalité par la description sensible d'une heure décisive :

L'essence de la nouvelle, c'est en quelques mots la vie d'un homme exprimée par la force infiniment sensible de l'heure où se joue son destin. La différence d'étendue de la nouvelle et du roman ne fait que symboliser [...] le fait que le roman restitue aussi dans son contenu la totalité d'une vie, en plaçant l'homme et son destin dans la pleine profusion de tout un univers, et que la nouvelle ne remplit cette fonction que d'un point de vue formel, en façonnant un épisode vécu de manière si sensible qu’à côté de sa portée universelle tous les autres moments de l'existence deviennent superflus ${ }^{18}$.

Dix ans plus tôt, en I90I, paraît Buddenbrooks, le roman qui vaudra à Thomas Mann, mais bien plus tard (en 1929!) le Prix Nobel. Le roman moderne de langue allemande ne s'impose qu'au $x^{\mathrm{e}}$ siècle sur le plan international, avec Thomas Mann, Stefan Zweig, Robert Musil, Kafka et, après la guerre, Heinrich Böll et Günter Grass. Tous ces auteurs ont écrit des nouvelles. Les romans allemands du XIX ${ }^{e}$ siècle, nombreux, sont beaucoup moins lus en Allemagne même, à l'exception peut-être de ceux du Prussien Theodor Fontane, qui fut aussi un grand nouvelliste. Quelques œuvres du tout début du XIX ${ }^{\mathrm{e}}$ siècle, parmi lesquelles figurent le roman Les Affinités électives, œuvre conçue au départ pour être une nouvelle, et les romans de Hölderlin, de Novalis et de Tieck, ont marqué l'histoire de la littérature à défaut de rencontrer vraiment les faveurs du public. Les lycéens allemands étudient les grands nouvellistes, le Prussien Ernst Theodor Amadeus Hoffmann, le Silésien romantique Joseph von Eichendorff, le Hessois Georg Büchner (Lenz), l'Allemand du Nord Theodor Storm, les Suisses Conrad Ferdinand Meyer et Gottfried Keller (qui a aussi écrit un roman), les Autrichiens Adalbert Stifter et Franz Grillparzer. Cette prédominance de la nouvelle sur le roman ou, autrement dit, l'absence d'un Balzac, d'un Dickens, d'un Stendhal ou d'un Zola, s'expliquent, selon nous, aussi par l'éclatement de l'Allemagne, par l'absence d'une "totalité»

I8. "Das Wesen der Novellenform ist kurz gefaßt: ein Menschenleben durch die unendlich sinnliche Kraft einer Schicksalsstunde ausgedrückt. Der Unterschied der Ausdehnung der Novelle und des Romans ist nur ein Symbol [...] dessen, daß der Roman die Totalität des Lebens auch inhaltlich gibt, indem er den Menschen und sein Schicksal in den vollen Reichtum einer ganzen Welt hineinstellt, die Novelle dies aber nur formal tut, durch eine derart stark sinnliche Gestaltung einer Episode des Lebens, daß neben deren Allumfassen alle andern Teile des Lebens überflüssig werden.» (Georg Lukács, «Bürgerlichkeit und l'art pour l'art: Theodor Storm ", Die Seele und die Formen, Berlin, E. Fleischel, I9II, p. I57 et suiv.) 
politique comparable à celles de Grande-Bretagne ou de France, les patries du roman moderne. D'une certaine manière, Buddenbrooks exprime la fin de cette Allemagne éclatée et la naissance (douloureuse) d'un pays unifié et modernisé : à la tonalité critique et nostalgique des nouvelles de l'Allemagne encore éclatée du XIX ${ }^{\mathrm{e}}$ siècle et de leur univers étroit succède le roman en tant qu'expression de la perte de cet univers. Dans le cas du roman allemand, $l^{\prime}$ " absence de domicile transcendantal ${ }^{19}$ ", concept forgé au début du $\mathrm{xx}^{\mathrm{e}}$ siècle par Georges Lukács dans La Théorie du roman, est aussi l'absence d'une patrie politique, culturelle et géographique aux contours bien définis, d'où sans doute une difficulté bien allemande (et autrichienne) à imposer le roman comme genre susceptible de décrire et de penser la société dans son ensemble, problème auquel était déjà confronté Goethe, qui recourait volontiers à un discours symbolique, comme on l'a vu. Rien d'étonnant donc à ce que le roman allemand se soit surtout illustré dans les genres historique et critique, voire "symbolique» et d'une certaine manière "antiréaliste», si l'on pense à Kafka, dans un empire austro-hongrois à soi-même opaque. Les grandes nouvelles allemandes du $\mathrm{XIX}^{\mathrm{e}}$ siècle restent une richesse à découvrir pour les publics étrangers et ont fondé une tradition qui a résisté au roman et survécu à la mode passagère de la short story, en vogue après 1945 .

Encore aujourd'hui la nouvelle s'affirme dans la sphère germanophone au point d'occuper les premières places dans les classements des meilleures ventes, soit des recueils de récits brefs ${ }^{20}$, soit, plus souvent, des récits longs, de La Découverte de la saucisse au curry d'Uwe Timm en 1993 à En crabe de Günter Grass en 2002 et Minute de silence de Siegfried Lenz en 2008. Ce fait est d'autant plus remarquable que les supports traditionnels de la nouvelle et des récits brefs, à savoir les périodiques littéraires, les revues, les magazines, ont ou disparu ou cessé de jouer un rôle économique de premier plan dans le champ littéraire. Sur le plan structurel, la nouvelle allemande reste proche des définitions du XIX ${ }^{\mathrm{e}}$ siècle, une ligne narrative simplifiée, peu de personnages et un tournant ou un "faucon", un élément qui distingue le texte de tout autre. Le fait que des romanciers allemands affectent d'honorer le genre "inférieur" de la nouvelle est un hommage assez mérité, car le roman, qui renonce de plus en plus à représenter «la

19. Georg Lukács, Die Theorie des Romans ( $\mathrm{I}^{\mathrm{re}}$ éd. I916), 2000, dtv, p. 32 : « [...] die Form des Romans ist, wie keine andere, der Ausdruck der transzendentalen Obdachlosigkeit [...].» («[...] la forme romanesque exprime plus que nulle autre l'absence de domicile transcendantal [...].")

20. Par exemple Récits sans gravité. Histoires de la province est-allemande (1999), roman d'Ingo Schulze qui se compose de 29 récits brefs, ou Mon siècle de Günter Grass (2000), qui se compose de cent récits. 
profusion de tout un univers» dont parlait Georges Lukács, a fini par se rapprocher de la nouvelle.

Cette convergence entre le roman et la nouvelle relègue Les Affinités électives et leur opposition entre roman «mortifere» et nouvelle "vitaliste» dans un passé révolu, celui de la "période artistique"(Kunstperiode ${ }^{21}$ ), un concept forgé par le poète Heinrich Heine pour désigner l'époque de Goethe, celle où l'appréhension artistique du réel dominait encore et pour la dernière fois toutes les autres. On ne demande plus, comme Goethe, à la nouvelle de démentir le mensonge du roman et de suggérer, par cette double négation, la vérité d'un autre roman. Le roman actuel, dans sa veine "réaliste», doit tenir compte des connaissances accumulées depuis le $\mathrm{XIX}^{\mathrm{e}}$ siècle et de l'évolution des mœurs qui s'en est suivie, sauf à s'exposer au reproche ne pas être à la hauteur du sujet traité : c'est bien la «scientificité» que Heine pointait dès I828 qui l'a emporté22. Le roman et la nouvelle sont désormais dépendants des connaissances acquises, notamment dans «leurs» domaines traditionnels, les sciences sociales et humaines. Ils sont en outre concurrencés par des arts qui procurent des émotions plus fortes et plus immédiates, tel le cinéma au sens large du terme. Genres à l'origine "raisonnables», tentant de réfléchir à la place de l'homme dans le monde, le roman et la nouvelle ne sont plus le «moyen de connaissance» dont on rêva si souvent aux $\mathrm{XIX}^{\mathrm{e}}$ et $\mathrm{XX}^{\mathrm{e}}$ siècles et ne font plus au mieux que «jouer» le rôle qu'ils ont perdu dans une époque qui se passe d'eux pour s'informer, réfléchir ou transformer le réel en expérience esthétique. Si l'interprétation des fictions du passé représente encore un moyen légitime et indispensable de comprendre la vision du monde propre à une époque, aujourd'hui plus que jamais on peut donner raison à Hegel qui affirmait dans son cours d'esthétique : "Pour nous l'art n'est plus le moyen suprême par lequel la vérité manifeste son existence ${ }^{23} \ldots »$

2I. Le terme apparaît pour la première fois dans la critique que Heinrich Heine fait paraître en I828 à propos des deux volumes de Wolfgang Menzel intitulés Die deutsche Literatur: Sämtliche Schriften, éd. Klaus Briegleb, Munich, Hanser, I976, t. I, p. 444.

22. Ibid., p. 444.

23. G. F. W. Hegel : «Uns gilt die Kunst nicht mehr als die höchste Weise, in welcher die Wahrheit sich Existenz verschafft..." (Ästhetik, [note 7], p. I69.) 
